\section{P 30 DELIVERING SAFE OPIATE INFUSIONS FOR ANALGESIA IN CHILDREN}

Asia N Rashed, ${ }^{1}$ Cate Whittlesea, ${ }^{2}$ Virginia Aguado, ${ }^{1}$ Ben Forbes, ${ }^{3}$

Stephen Tomlin'. 'Evelina London Children's Hospital, Guy's \& St Thomas' NHS

Foundation Trust; ${ }^{2}$ Durham University; ${ }^{3}$ King's College London, King's Health

Partners

\subsection{6/archdischild-2015-308634.38}

Aim Opiate infusions are essential therapy for children with severe pain, but have been associated with serious medication errors. ${ }^{1-4}$ These infusions require complex dose calculations, multiple manipulations and complicated administration rate adjustments. The project aimed to minimise the risk of medication errors in nurse- or patient-controlled analgesia (N/PCA) for children.

Method A system management approach was developed to minimise complex calculations and individualised medicine manufacture by providing standard dose-banded concentrations of morphine infusion, manufactured aseptically, for N/PCA administration using pre-programmed safety pumps. The new system was implemented in three phases:

1. Pre-implementation: Direct observation in theatres and wards of current practice; analysis of individually prepared syringes; focus groups (ward nurses, recovery nurses, and anaesthetists) on the current practice; Product design: packaging and labelling; Failure mode $\&$ effect analysis.

2. Implementation: Reconfiguration of the smart pumps with the new morphine standardised concentrations protocols; production of new colour-coded protocols, labels and standard operating procedures for ward/theatres use; evaluation of storage of the new product in different areas.

3. Post-implementation: direct observation of administration process of morphine standardised prefilled syringe (PFS); focus groups on the new system (morphine PFS); 6-month follow up study after full roll out.

Results Observation of morphine infusions (153) prepared by healthcare professionals for 128 children (7.5 \pm 5.6 years; mean $\pm S D$ ) identified major differences in preparation methods. Analysis of the individually prepared infusions (78) identified that $61.5 \%$ deviated from the target concentration by greater than the pharmacopoeial limit $( \pm 7.5 \%)$, of which $75 \%(36 / 48)$ were in excess with $28 \%(10 / 36)$ deviating by more than $+20 \%$. A variety of preparation practices led to the out-of-specification results, including lack of appreciation of the overage in morphine ampoules; "Say I've got $27 \mathrm{~kg}$ child, and you have to put $27 \mathrm{mg}$ in. I'll always put the $20 \mathrm{mg}$, all of the ampoules in, as I opposed to drawing it up in a syringe I will be losing some and I want to make sure that I get what I think in my head is 27 , so the first 2 will get the full ampoule and only the last $7 \mathrm{mg}$ that I will draw up in a $1 \mathrm{~mL}$ syringe." (Anaesthetist). Three standardised concentrations of morphine were established:

$3 \mathrm{mg} / 50 \mathrm{~mL}$ glucose $5 \%$ for weight $\leq 3.99 \mathrm{~kg}$

$10 \mathrm{mg} / 50 \mathrm{~mL}$ sodium chloride $0.9 \%$ for weight $\geq 4 \mathrm{~kg}-19.9 \mathrm{~kg}$

$50 \mathrm{mg} / 50 \mathrm{~mL}$ sodium chloride $0.9 \%$ for weight $\geq 20 \mathrm{~kg}$

Pilot implementation of standardised morphine infusions $(35$ to date) was well-received by anaesthetists and nurses. Colour coded protocols and labels were regarded as lowering the risk of wrong selection.

Conclusion The project addressed the unacceptable variation identified in individually prepared medicines and simplified their administration. Whilst it is known that standard concentration morphine infusions can be utilised in paediatrics, we have demonstrated for the first time that it is possible to use them for N/PCA. Limits of accuracy for the pumps were quantified and accommodated in defining the weight-concentration relationships to verify reliable delivery of continuous infusions and boluses.

\section{REFERENCES}

1 Rashed AN, Wong ICK, Cranswick N, et al. Adverse Drug Reactions in ChildrenInternational Surveillance and Evaluation (ADVISE): a multicentre cohort study. Drug Saf 2012;35:481-94.

2 Rashed AN, Wong ICK, Cranswick N, et al. Risk factors associated with adverse drug reactions in hospitalised children: international multicentre study. Eur J Clin Pharmacol 2012;68:801-10.

3 Rashed AN, Neubert A, Tomlin S, et al. Epidemiology and potential associated risk factors of drug-related problems in hospitalised children in United Kingdom and Saudi Arabia. Eur J Clin Pharmaco 12012;68:1657-66.

4 Neubert A, Verhamme K, Murray ML, et al. The prescribing of analgesics and non-steroidal anti-inflammatory drugs in paediatric primary care in the UK, Italy and the Netherlands. Pharmacol Res 2010;62:243-48. 